\title{
A Preliminary User Interface Study of Speech Enhancement System
}

\author{
Kenji Matsui ${ }^{\text {a, }}$, Yuma Otsuki ${ }^{\mathrm{a},}$, Yoshihisa NAKATOH ${ }^{\mathrm{b}}$, Yumiko O. Kato ${ }^{\mathrm{c}}$ \\ ${ }^{a}$ Osaka Institute of Technology, 5-16-1,Omiya,Asashi-ku, Osaka 535-8585, Japan \\ ${ }^{b}$ Kyushu Institute of Technology, 1-1 Sensui-cho, Tobata-ku, Kitakyushu 804-8550, Japan \\ ${ }^{c}$ St. Marianna University School of Medicine, 2-16-1 Sugao, Miyamae-ku, Kawasaki 216-8511, Japan \\ *Corresponding Author: matsui@elc.oit.ac.jp
}

\begin{abstract}
The feasibility of using a motion sensor to replace a conventional electrolarynx(EL) user interface was explored. Forearm motion signals from MEMS accelerometer was used to provide on/off and pitch frequency control. The vibration device was placed against the throat using support bandage. Very small battery operated ARM-based control unit was developed and placed on the wrist. The control unit can convert the tilt angle into the pitch frequency. Speech generation was tested with various forearm movements, and then a simple and small action was chosen to control the device. To accomplish stable and practical speech generation, device enable/disable function and pitch range adjustment functions were also added. A simple comparison study has been made with three well-trained normal speakers. Results of the study showed that the prototype system was able to produce the pitch patterns similar to those in natural utterances.
\end{abstract}

Keywords: Electrolarynx, MEMS accelerometer, hands free

\section{Introduction}

People who have had laryngectomies have several options for the restoration of speech, but no currently available device is satisfactory. The artificial larynx, typically a hand-held device which introduces a source vibration into the vocal tract by vibrating the external walls, is the easiest for patients to master, but does not produce airflow, so the intelligibility of consonants is diminished and the speech is uttered at a monotone frequency. Alternatively, esophageal speech does not require any special equipment, but requires speakers to insufflate, or inject air into the esophagus, and limits the pitch range and intensity. Both esophageal speech and tracheo-esophageal speech are characterized by low average pitch frequency, large cycle-to-cycle perturbations in pitch frequencies, and low average intensity. As for utilizing esophageal speech, it was found that age was the important factor. When laryngectomized patients get older, they face difficulty in mastering the esophageal speech or keep using esophageal speech because of the waning strength. For that reason, the electrolarynx is an important device even for the people who use esophageal speech.

As for the advantages of EL, firstly, one can speak in long sentences that are easily understood. Secondly, no special care requirements are needed; the EL only has to be placed up against the neck and turned on. Thirdly, the EL can be used by almost everybody, regardless of the post-operative changes in the neck. In those few cases where scarring prevents proper placement of the EL, an intraoral version can be used.

On the other hand, there are a couple of disadvantages. Firstly, the EL has a very mechanical tone that does not sound natural. There usually is little change in pitch or modulation. Secondly, one must use their hand to control the EL all the time, and its appearance is far from normal.

An EL system that has a hands free user interface could be useful for enhancing communication by alaryngeal talkers, especially in hands busy environments. Also, the appearance can be almost normal even though the system requires slight hand movement. Almost all people frequently use gestures when they talk. It would be quite convenient if the EL users could utilize gestures to control the device because of its hands free features. Furthermore, gesture control has a lot of potential to control not only just on/off function, but also many other various functions. Pitch frequency control is one of the important mechanisms for EL users to be able to generate naturally sounding speech. There are many studies of pitch controlling methods (1), (2), (3). In fact, a couple of EL devices with 
pitch control mechanism are commercially available now (4), (5). However, none are hands free.

The present study was undertaken to explore the feasibility of using gesture control method to replace the conventional EL user interface in terms of both on/off function and pitch control. Also, a wrist-watch type EL control device was designed and evaluated in order to determine the actual speech generation performance in a real environment. The specific goals were: 1) to determine the practical hands free user interface method for EL system, and 2) to determine whether the generated speech has high intelligibility and naturalness.

\section{Determination of Needs - Survey Results}

\subsection{User Profile}

A set of techniques - including user observations, interviews, and questionnaires - were used to understand implicit user needs. As for the questionnaire survey, the total number of laryngectomized participants was $121(87 \%$ male, $13 \%$ female), including $65 \%$ esophageal talkers, $12 \%$ EL users, $7 \%$ both, and $21 \%$ used writing messages to communicate.

\subsection{Survey Results}

Almost all of the participants claimed that most public areas are difficult for oral communication due to the noisy environment. Typical public areas include train stations, inside of train cars, inside of vehicles, restaurants/pubs, and conventions/gatherings. Some of the needs confirmed from the survey are:

- Naturally sounding voice, not like mechanical tone

- $\quad$ Light weight device

- Smaller device, low profile

- Hands-free, easy to use

- Low cost

Based on the survey results, the present study was conducted to meet the essential user needs.

\section{Hands Free UI Design}

\subsection{Gesture Control}

Gesture control UI can be developed through the use of a system based on photo detector, camera, or accelerometer. Based on the survey results, a3-axis MEMS accelerometer was used in this study. MEMS sensors are very small, low cost, and a fit the system requirements well.

\subsection{Pitch Control}

A MEMS accelerometer accurately measures acceleration, tilt, shock and vibration in applications. The challenge in designing the pitch control algorithm that use a MEMS accelerometer output to control pitch contour is to reconcile the numerical ranges between two types of data. MEMS output bytes are integers in the range -128 to 127 for a range of $\pm 2 \mathrm{G}$. Often this issue can be easily reconciled by linear mapping of one range of values (such as MEMS data values -128 to 127 ) into another range (such as 67 to 205 expected as the typical male pitch range).

Another possible pitch control method is to utilize a pitch contour generation model, such as Fujisaki's model (6). The system needs to have a strategy to extract both the phrase component and the accent component from the MEMS output. The model based method is easier to generate relatively stable pitch contour, however, it may lose some flexibility to generate various pitch patterns.

In this study, the simple linear mapping method was used to evaluate the pitch control performance. However, we plan to run the comparison study between the linear mapping method and the model-based method as one of our next phase tasks.

\section{System Implementation}

\subsection{Hardware System Design}

The pitch control algorithm described above was implemented on a small ARM cortex-M0 CPU board. A block diagram of the hardware architecture is shown in Fig. 1. EL transducer with neck-bandage has also been prepared to place it to the optimal location on the neck. We introduced the ARM board in order to meet the user requirements, i.e. small, comfortable weight, and low cost. The ARM-based hardware unit consists of a small board $(34 \mathrm{~mm} \times 34 \mathrm{~mm})$ with a $48 \mathrm{MHz} \mathrm{C} 1114$, a 32 bit ARM cortex-M0, a ten bit PWM with $10 \mathrm{kHz}$ sampling rate, a USB interface, $32 \mathrm{kB}$ FLASH memory, and three $1.5 \mathrm{~V}$ batteries. Picture 1 shows the ARM unit and the EL transducer.

\subsection{Pitch Control}

Hand gestures are a very important part of language. A preliminary UI study using forearm movement was conducted in order to evaluate feasibility of the pitch control mechanism. Fig.2 shows the forearm tilt and the MEMS output (x-axis) when the controller was placed on 


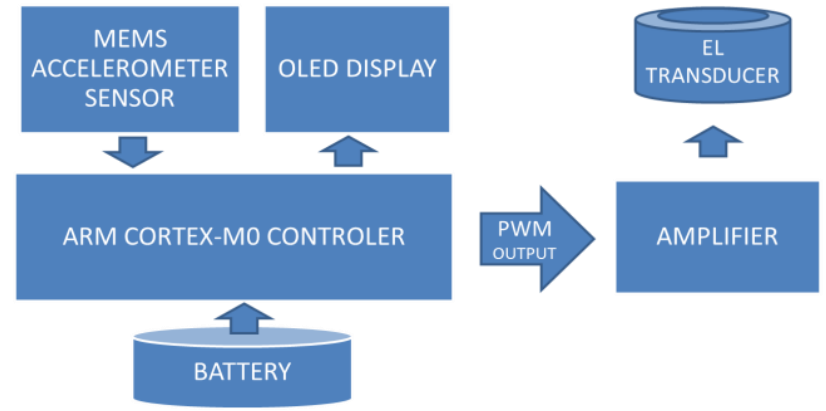

Fig. 1. Block Diagram of the Hardware Architecture

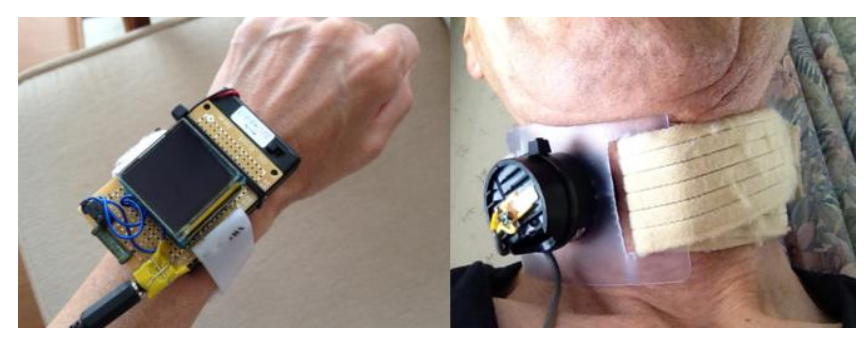

Picture 1. EL Controller and Transducer

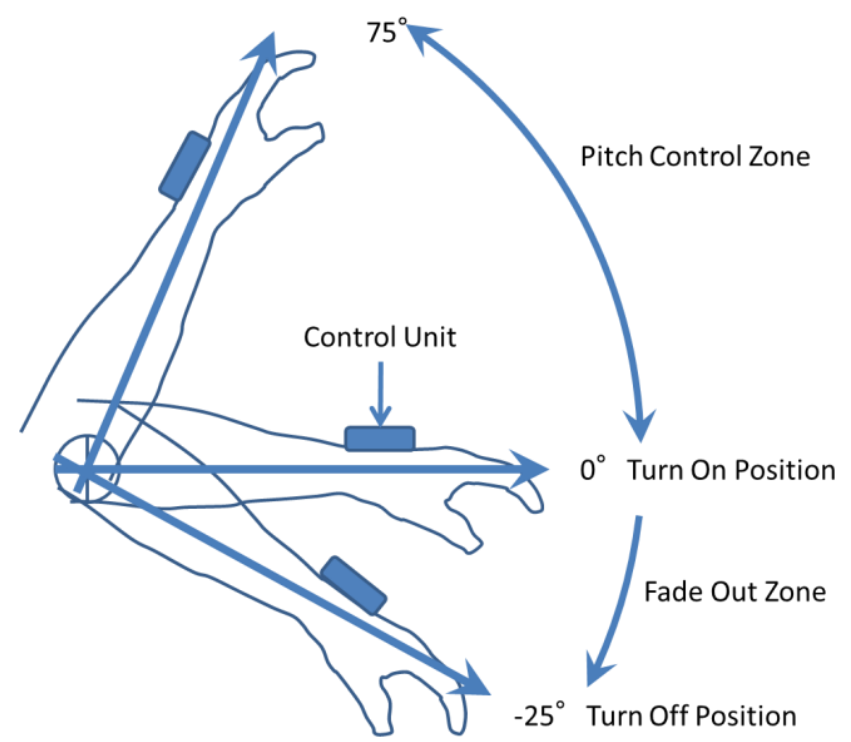

Fig. 2. Forearm Tilt and Pitch Control

the wrist. From the horizontal position $\left(0^{\circ}\right)$ to the $75^{\circ}$ upward position is the normal pitch control zone. From the horizontal position to the $-25^{\circ}$ downward position is the fading out zone, where phrase ending pitch pattern is adjusted based on the forearm moving speed. As for the conversion from the MEMS output to the pitch frequency, there are four pitch ranges. Fig. 3 shows the relation

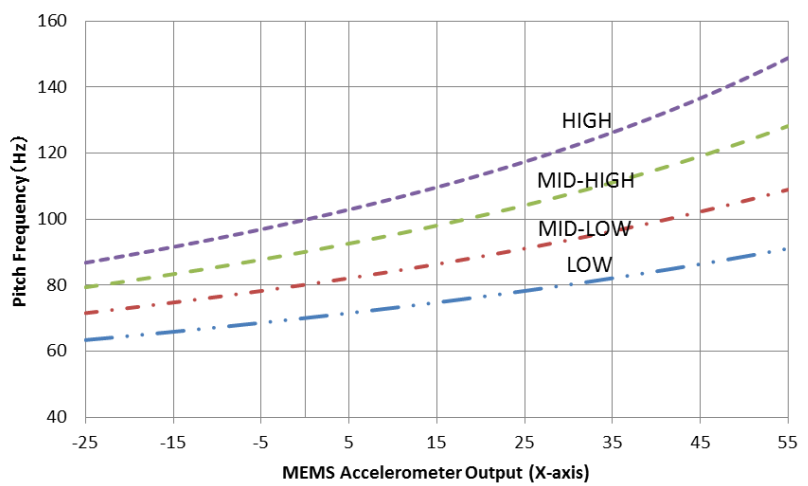

Fig. 3. Relation between MEMS Output and Pitch

between the MEMS output and the four ranges of pitch frequency, i.e. high, mid-high, mid-low, and low. Users can select one of the four ranges.

\section{$4.3 \quad$ ON/OFF Control}

Reliable EL ON/OFF control is very important for users to talk comfortably. As you can see in Fig.2, EL vibrates at the normal pitch control zone, i.e. from $0^{\circ}$ position and higher. EL stops the vibration at the $-25^{\circ}$ position or lower. The hysteresis is necessary to avoid unstable behavior near the on/off threshold. If the phrase does not have an accent, the pitch rises from a low starting point on the first mora, and then levels out. Such pitch contour is generated by moving the forearm downward very quickly. However, most of the accented phrases are generated by gradual movement.

\subsection{LOCK Mechanism}

It is very important to enable/disable the controller easily and quickly while users are wearing the device. Y-axis output of the MEMS accelerometer was used to implement such a lock mechanism. By twisting the wrist quickly and generating $2 \mathrm{G}$ acceleration, the user can enable/disable the EL.

\section{Evaluation}

A preliminary usability test was performed. Three normal speakers participated in this study. After 30 minutes of practice, 17 test sentences were pronounced using their own voice and using the prototype. Then, the pitch contours were analyzed. Fig.4 shows one of the comparison test results. The comparison study showed that the overall pitch trends were similar, however, more practice is necessary 

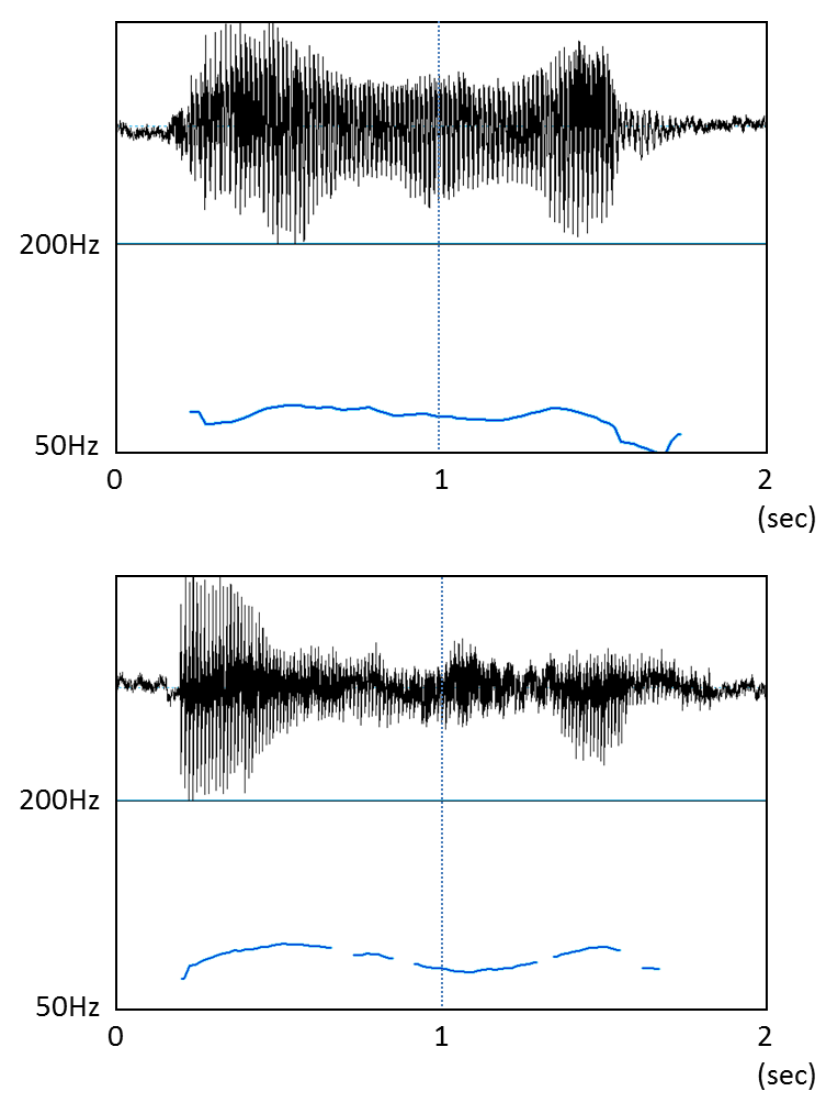

Fig. 4. EL speech output and pitch contour (below) in comparison with natural speech and pitch contour (above). Both phrases are "aoi o ueru".

to control the pitch precisely.

\section{Discussion}

The results of this study indicate that the usability of EL speakers could be improved by MEMS accelerometer based hands free UI controller. The ability to control the pitch contour of EL speech with the proposed linear mapping method implies that hand gesture control may be adequate for implementation of the hands free user interface for EL device. A model-based pitch generation study will be tested as the next step. A learning effect was observed even during the short preliminary test. A more detailed and precise study in terms of the learning curve has to be performed. As for the gesture control, we tested only the forearm movement, however, it is necessary to test other body locations where users might be able to control the EL device more easily and naturally. According to the user requirements, the evaluation of appearance also needs to be considered. In the study, we set a relatively narrow pitch range in order to avoid wild swings in pitch. A better pitch control range needs to be investigated.

\section{Conclusion}

MEMS accelerometer based hands free UI for EL device was proposed, and a hand gesture control unit was designed. Results of the preliminary evaluation indicated that the proposed method has a potential to make the EL output prosody more natural, easy to use, and less distinct appearance. However, users may be required to undergo some training before utilizing the device comfortably. The device also needs further evaluation in terms of how to generate the pitch, where to wear the device, and how to make the device less conspicuous.

\section{Acknowledgment}

This work was supported by JSPS KAKENHI Grant-in-Aid for Scientific Research(C) Grant Number 24500664.

\section{References}

(1) K. Matsui, "A preliminary user interface study of speech enhancement system", Proc. Spring Meet. Acoust. Soc. Jpn, 455-456 (2012)

(2) Kikuchi, Y., and Kasuya, H. : "Development and evaluation of pitch adjustable electrolarynx", In SP-2004, 761-764, 2004

(3) Takahashi, H.,Nakao, M., Ohkusa, T., Hatamura, Y., Kikuchi, Y., and Kaga, K., 2001. Pitch control with finger pressure for electrolaryngial or intra-mouth vibrating speech.Jp. J. Logopedics and Phoniatrics, 42(1), 1-8.

(4) SECOM company Ltd., Electrolarynx "MY VOICE", (http://www.secom.co.jp/personal/medical/myvoice.ht $\underline{\mathrm{ml}})$

(5) Griffin laboratories, TruTone users guide.

(6) H. Fujisaki, In Vocal Physiology: Voice Production, Mechanisms and Functions, Raven Press, 1988 